\title{
Cryo-EM and Molecular Biology Approaches for Characterizing Flagellar Filament Structures of Caulobacter crescentus
}

Juan Sanchez ${ }^{1}$, Nicoleta Ploscariu ${ }^{1}$, Eric Montemayor ${ }^{1}$, Daniel Parrell ${ }^{1}$, Gan Li ${ }^{1}$, Rebecca Dillard ${ }^{2}$, Conrad Shebelut ${ }^{2}$ and Elizabeth Wright ${ }^{1}$

${ }^{1}$ University of Wisconsin - Madison, Madison, Wisconsin, United States, ${ }^{2}$ Emory University, Atlanta, Georgia, United States

Bacteriophage adsorption and bacterial cell mobility are both dependent upon flagella, massive oligomeric assemblies of individual flagellin proteins that protrude from bacteria and can span several $\mu \mathrm{m}$ in length. In Caulobacter crescentus, a gram-negative alpha-proteobacterium that commonly lives in oligotrophic fresh and salt-water environments, the flagellar filament is composed of six flagellin proteins, fljJ, fljK, fljL, fljM, fljN, and fljO, which exhibit significant amino acid sequence similarity [1]. These individual flagellins self-assemble into compositionally and conformationally complex flagella structures with variable curvature. A recent model proposed that filament curvature is due to polymorphic switching, where shorter inter-protofilament spacings are found on one side of the curved flagellin and longer interprotofilament spacings are found on the opposite side of the flagellin [2]. This composition leads to the periodic waveform observed in flagellar filaments (Figure 1A), which precludes high-resolution structure determination of flagella via single particle helical reconstruction methods. To overcome this issue, genetic disruption of all but one flagellin gene was combined with random mutagenesis to create straightened flagella in Caulobacter crescentus. We used motility assays, fluorescence light microscopy and negative stain transmission electron microscopy (TEM) to identify strains with "straightened" flagellins that are amenable to high resolution structure determination via cryo-electron microscopy (cryoEM).

A compositionally pure fljK flagella with a single point mutation to confer straightening was extracted from cell bodies via low speed centrifugation, then the detached flagella were separated from other proteins via high speed centrifugation. Purified flagella samples were plunge frozen onto glow-discharged, 200 mesh copper Quantifoil grids (Quantifoil, Germany) in liquid ethane using a Vitrobot Mark IV (FEI, Hillsboro, Oregon). Vitrified grids were imaged on a Titan Krios cryo-electron microscope operated at $300 \mathrm{kV}$. Micrographs were recorded on a Gatan K3 camera in counting mode without use of an energy filter at a pixel size of $0.87 \AA$ and a total dose of $\sim 60 \mathrm{e}^{-} / \AA^{2}$ fractionated over 45 images per exposure (Figure 1B). Motion correction and all steps for 3-D reconstruction were performed in Relion 3.0, using a manually curated subset of 643 micrographs and Relion's helical reconstruction pipeline [3, 4]. Manual helical particle picking with a box size of 360 pixels and rise of $30 \AA$ resulted in 67,894 particles that were subjected to 2-D classification with 3-fold binning, resulting in a stack of 65,568 particles (Figure 2A). $A b$ inito model generation was accomplished by taking a $20 \AA$ low-pass filtered model of the B. subtilis flagellin [2] and performing helical 3-D classification with two-fold binning of particles in Relion with an initial rise and twist value of $65^{\circ}$ and $4.6 \AA$, respectively. A single class was obtained with clear secondary structure features and a parameterized rise and twist of $65.2^{\circ}$ and $4.9 \AA$, respectively. This volume was used then used as the starting model for automated refinement in Relion using a Z-height of 80\%, followed by masking with a soft edge and per-particle CTF estimation and motion correction, and unbinning to the native pixel size of $87 \AA$. The resulting volume exhibited clearly defined mainchain and sidechain features. An estimated B-factor of -55 was used for map sharpening in Relion. The half-sets used for the final reconstruction exhibited a $\mathrm{FSC}_{0.143}$ crossover at $3.4 \AA$. Iterative rounds of manual model building and automated refinement were conducted in Coot and Phenix, respectively [5, 6]. 
Our structure of the FljK flagella (Figure 2B) provides a tracible platform for rationally designing other flagellins, such as FljL, which will subsequently facilitate structure determination of other flagella and allow comparison of similar yet distinct components of flagella in Caulobacter crescentus. These studies are essential for understanding flagella composition and function, which may lead to the development and engineering of novel antimicrobials aimed at inhibiting multi-flagellin bacteria [7].
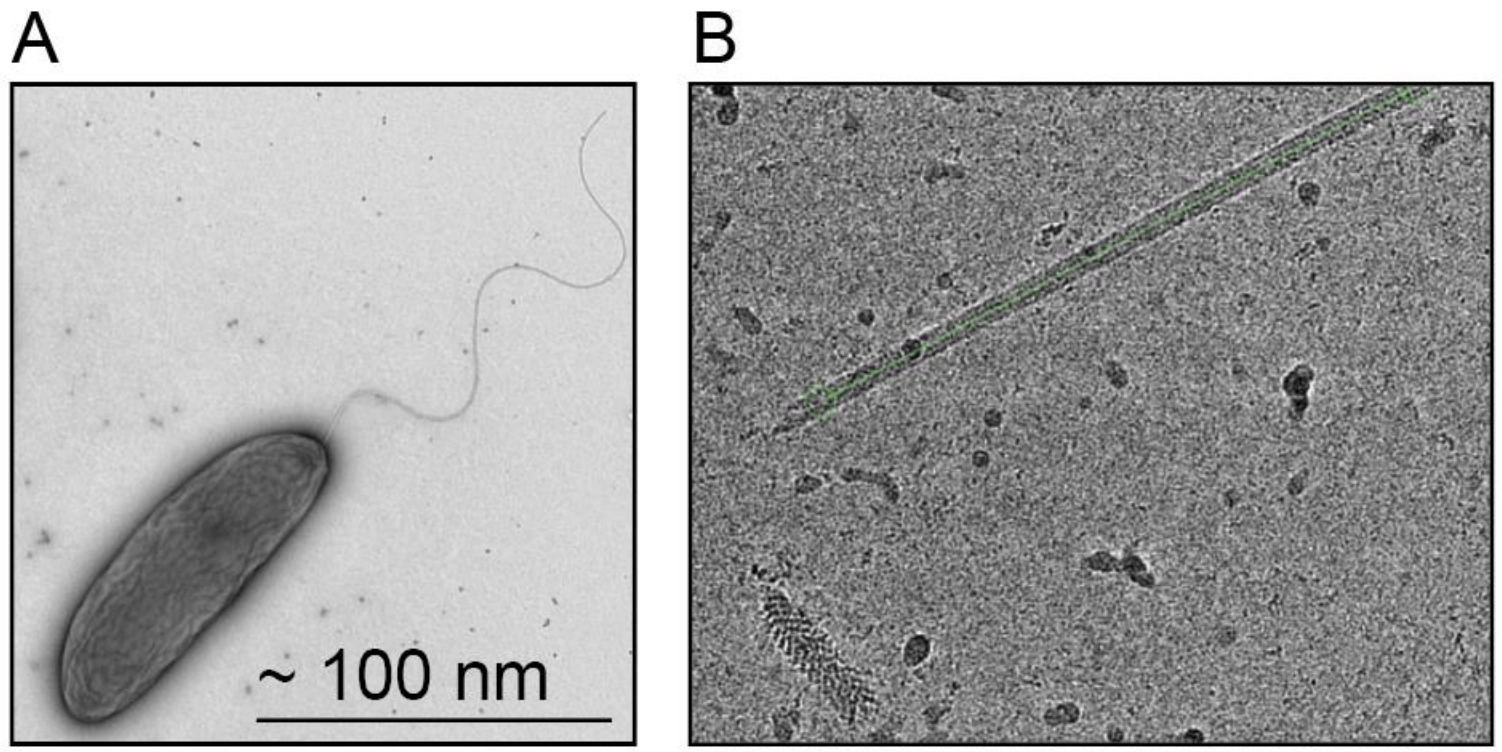

Figure 1. Caulobacter crescentus flagella characterization. A) Non-straightened flagella. B) Cryoelectron micrograph of straightened $\mathrm{FljK}$. The micrograph, which has been motion corrected to compensate for beam induced movement, has an estimated defocus of $\sim-2 \mu \mathrm{m}$ and is rendered with a lowpass filter of $10 \AA$. A hook structure, which is present in our preparations of FljK, is visible in the bottom left region of the micrograph.

A

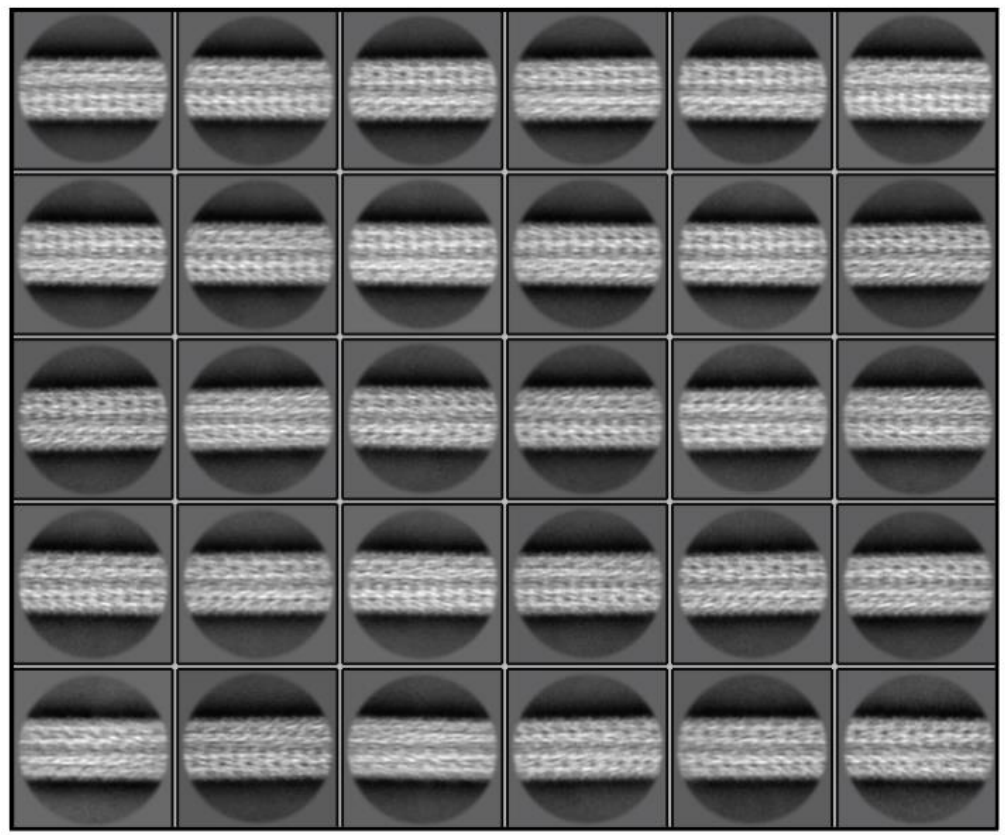

$\mathrm{B}$

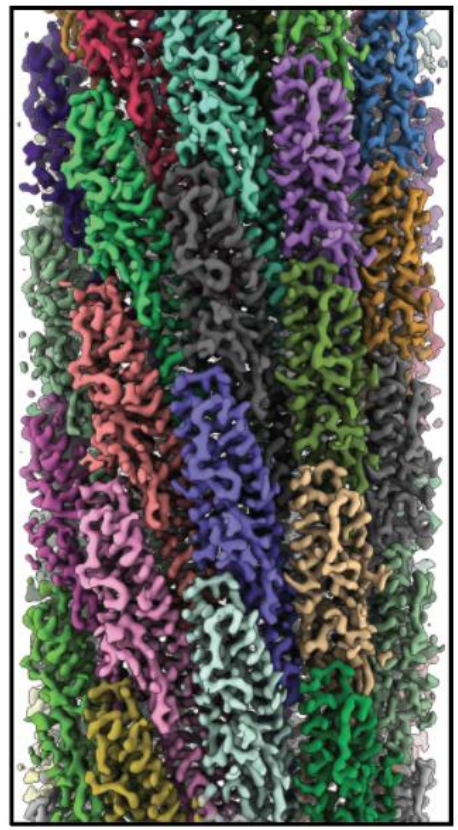


Figure 2. Structure of FljK from Caulobacter crescentus. A) 2-D classes generated in Relion 3.0. B) Final map derived from a map with a FSC0.143 crossover at $3.4 \AA$.

\section{References}

[1] A. Faulds-Pain, C. Birchall, C. Aldridge, et al., Flagellin redundancy in Caulobacter crescentus and its implications for flagellar filament assembly, J Bacteriol 193(11) (2011) 2695-707.

[2] F. Wang, A.M. Burrage, S. Postel, et al., A structural model of flagellar filament switching across multiple bacterial species, Nat Commun 8(1) (2017) 960.

[3] S. He, S.H.W. Scheres, Helical reconstruction in RELION, J Struct Biol 198(3) (2017) 163-176.

[4] J. Zivanov, T. Nakane, B.O. Forsberg, et al., New tools for automated high-resolution cryo-EM structure determination in RELION-3, Elife 7 (2018).

[5] A. Casanal, B. Lohkamp, P. Emsley, Current Developments in Coot for Macromolecular Model Building of Electron Cryo-microscopy and Crystallographic Data, Protein Sci (2019).

[6] D. Liebschner, P.V. Afonine, M.L. Baker, et al., Macromolecular structure determination using Xrays, neutrons and electrons: recent developments in Phenix, Acta Crystallogr D Struct Biol 75(Pt 10) (2019) 861-877.

[7] This research was supported in part by funds from the University of Wisconsin, Madison, Emory University, Children's Healthcare of Atlanta, the Emory Center for AIDS Research, the Georgia Research Alliance, Human Frontiers Science Program, National Institutes of Health (R01GM104540 and R01GM104540-03S1), and the National Science Foundation (0923395) to E.R.W. Cryo-EM data collection using the SECM resource at Florida State University was supported by National Institutes of Health grant U24GM123456. The authors gratefully acknowledge use of facilities and instrumentation at the Emory University Robert P. Apkarian Integrated Electron Microscopy Core and at the UW-Madison Wisconsin Centers for Nanoscale Technology (wcnt.wisc.edu) partially supported by the NSF through the University of Wisconsin Materials Research Science and Engineering Center (DMR-1720415). 